\title{
Evaluación ex-post proyecto quinua y su impacto socioeconómico en las familias asociadas, Chimborazo - Ecuador
}

Ex-post evaluation of the quinua project and its socioeconomic impact on associated families, Chimborazo
- Ecuador

\author{
Marco Aníbal Vivar Arrieta ${ }^{1 *}$ (D), Lucy Viviana Sánchez Varela ${ }^{1}$
}

\section{RESUMEN}

La investigación analizó el grado de efectividad de las acciones emprendidas por la Corporación de Productores y Comercializadores Orgánicos "Bio Taita Chimborazo", en función de sus logros e impactos en el campo socioeconómico de sus asociados, mediante la comparación con personas no asociadas del mismo sector y actividad. La población de estudio estuvo conformada por 1286 productores; de los cuales se obtuvo una muestra de 296 productores. La metodología, previamente se identificó de indicadores socioeconómicos cuyo instrumentó fue la encuesta, como resultados se obtuvo que los ingresos provenientes del proyecto quinua son mejores en los asociados en un $28,07 \%$ respecto a los no asociados, el acceso a créditos en un $26,51 \%$, la percepción de mejora en ingreso es un $19,7 \%$ más alta en los asociados, el nivel de seguridad social es $32,65 \%$ mayor que los no asociados, las mejoras en habitabilidad varían entre un $2,89 \%$, y la organización de la producción se elevó en un 50,42\%. Además, se realizó una entrevista guiada y documentada a través de los informes de gestión en los cuales se establece un nivel de cumplimiento de validez del $92,86 \%$, basado en el índice de Pearson, mientras que la eficiencia alcanza el 83,33\% y la efectividad un $88,66 \%$. La pertinencia y la sostenibilidad se ubican al $100 \%$ debido a la naturaleza del proyecto. Los resultados obtenidos de la investigación apuntan a un desarrollo socioeconómico discreto que, si bien existe, podría ser optimizado en función de sus asociados; las diferencias contrastadas entre asociados y no asociados se presentan en el plano económico, de vivienda y de productividad: los otros aspectos son comunes y no presentan diferencias significativas.

Palabras clave: estudio comparativo, indicadores socioeconómicos, COPROBICH, beneficio social, efectividad

\begin{abstract}
The research analyzed the degree of effectiveness of the actions undertaken by the Corporation of Organic Producers and Marketers "Bio Taita Chimborazo", according to its achievements and impact on the socio-economic field of its members, by comparing with non-associates in the same sector and activity. The study population consisted of 1286 producers; of which a sample of 296 producers was obtained. The methodology was previously identified from socioeconomic indicators which were instrumented by the survey. The results showed that the income from the quinoa project is $28.07 \%$ better for members than for non-members, access to credit is $26.51 \%$ better, the perception of improved income is $19.7 \%$ higher for members, the level of social security is $32.65 \%$ higher than for nonmembers, improvements in habitability vary between $2.89 \%$, and the organization of production has increased by $50.42 \%$. In addition, a guided and documented interview was conducted through the management reports in which a level of compliance validity of $92.86 \%$ was established, based on the Pearson index, while efficiency reached $83.33 \%$ and effectiveness $88.66 \%$. Relevance and sustainability are at $100 \%$ due to the nature of the project. The results obtained from the research point to a discrete socio-economic development which, although it exists, could be optimised according to its partners; the contrasting differences between partners and non-partners are in the economic, housing and productivity fields: the other aspects are common and do not present significant differences.
\end{abstract}

Keywords: comparative study, socioeconomic indicators, COPROBICH, social benefit, effectiveness

\footnotetext{
${ }^{1}$ Escuela Superior Politécnica de Chimborazo, Riobamba, Ecuador

*Autor de Correspondencia, e-mailmarcovivar6@hotmail.com
} 


\section{INTRODUCCIÓN}

El método de evaluación ex post ha sido considerado como una herramienta práctica de medición, de resultados especialmente adecuada para organizaciones de tipos social, en las que lo cuantitativo no es fundamental y más bien se tiende a la apreciación cualitativa como norma de medición de lo actuado (Medina, 2014). Complementando, la evaluación social o socio económica de proyectos, compara los beneficios con los costos que dichos proyectos implican para la sociedad (Contreras, 2014).

En esta última década, los gobiernos de los países en desarrollo han implementado nuevas orientaciones en cuanto a la transferencia de los fondos públicos, es así que se ha establecido un nuevo enfoque de desarrollo basado en la comunidad. Debido a este cambio se creó una metodología a cargo de la FAO (Organización de las Naciones Unidas para la Agricultura y la Alimentación), para formular y evaluar la manera en que se deben realizar las inversiones sociales y el impacto que estás ocasionando (FAO, 2015).

A nivel regional, la OEA (Organización de Estados Americanos), el ALBA (Alianza Bolivariana para América Latina y el Caribe) y la CELAC (Comunidad de Estados Americanos y Caribeños), entre otros, son organismos que miden socioeconómicamente las distintas sociedades con el fin de verificar si en los últimos años se ha logrado reducir la pobreza en América Latina, tomando en cuenta que los países siguen presentando altos niveles de marginación y rezago social (Fundación Ethos, 2015).

Ciertamente en el Ecuador, como una medida para mejorar la calidad de vida de los agricultores se ha fomentado la asociatividad de los habitantes rurales por medio de la conformación de asociaciones, corporaciones y fundaciones. Con el fin de aportar al desarrollo de la agricultura comercial y la agricultura familiar campesina, priorizando los servicios de comercialización, asociatividad e innovación, y con ello garantizar la soberanía alimentaria (MAGAP, 2017).

Con esta finalidad en Chimborazo se han creado organizaciones que impulsan la producción de Quinua como la Corporación de Productores y Comercializadores Orgánicos "Bio Taita Chimborazo" (COPROBICH), Escuelas Radiofónicas Populares del Ecuador (SUMAK LIFE) y Maquita Cushunchic $(\mathrm{MCCH})$, mismas que abarcan el $26 \%$ de la producción total del cereal, superado únicamente por Carchi con el 29\%, según datos INEC (Instituto Nacional de Estadística y Censos) (Montero, 2016).

En el año de 2003 se creó la Corporación de Productores y Comercializadores Orgánicos "Bio Taita Chimborazo" (COPROBICH) es una organización de productores legalmente reconocida de derecho privado, autónoma, sin fines de lucro y de servicio y beneficio social para sus socios que son indígenas. Desde su creación busca reducir la exclusión de los indígenas Puruhá de la provincia de Chimborazo a través de la valoración de la quinua, trigo, arroz de cebada y otros productos agropecuarios orgánicos (COPROBICH, 2017).

La organización ha recibido apoyo de diferentes instituciones públicas y privadas como el Ministerio de Agricultura y Ganadería, GAD Provincial de Chimborazo, GAD cantonal de Colta, Subsecretaria de Gestión de Riesgos (SGR), Escuelas Radiofónicas Populares (ERPE), TRIAS (ONG Bélgica), AVSF (ONG Francia), todos con la finalidad de apoyar y mejorar la cadena de valor de la quinua en todos sus eslabones (GADPC, 2017).

Por lo tanto, es importante realizar una evaluación ex post del impacto socioeconómico de las actividades desarrolladas por COPROBICH con las familias productoras de quinua del cantón Colta, provincia de Chimborazo, en el año 2017, mediante un estudio comparativo y contrastante entre asociados y no asociados, determinando el grado de efectividad de las acciones emprendidas en función de sus logros e impactos.

\section{MATERIALES Y MÉTODOS}

Se realizó una previa investigación documentada sobre la metodología a utilizarse, para realizar análisis valorativos de los programas y proyectos con el propósito de 
conocer si se logran los resultados esperados y cuál es el impacto que se genera en el mismo. Para ello se siguió, implemento y adapto el modelo de Navarro et al. (2016). Este diseño de evaluación, se aplica cuando se tiene una misma población con al menos dos medidas temporales (asociados y no asociados), lo que permitirá ser contrastada con los datos de las familias de los asociados con los no asociados, con el fin de verificar las diferencias permitiendo tener una idea clara de la tendencia.

\section{Selección de indicadores}

Los indicadores socioeconómicos (Figura 1) se obtuvieron mediante una encuesta (Apéndice 1) y una entrevista (Apéndice 2-3).

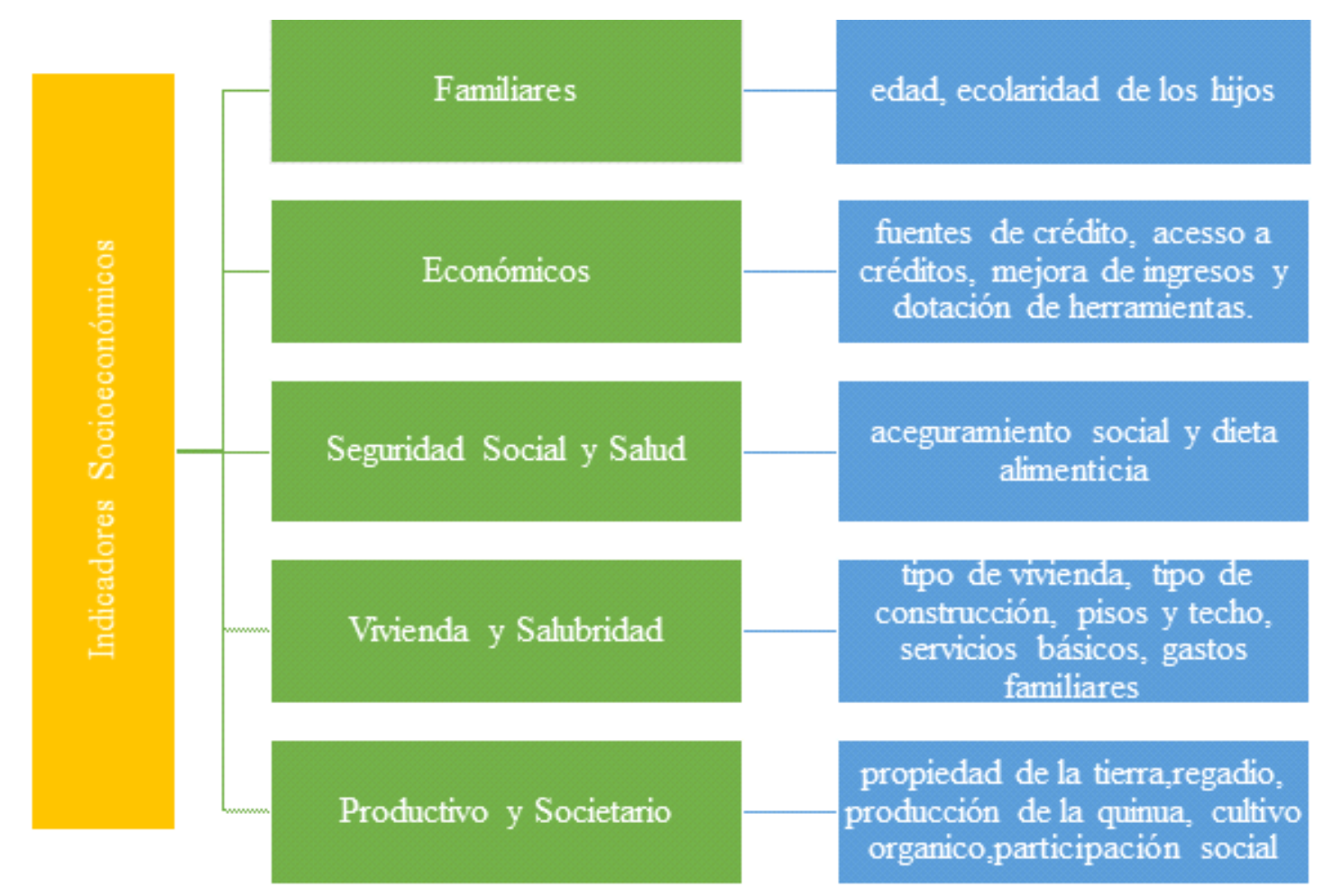

Figura 1. Indicadores socioeconómicos.

Posteriormente se realizó la validez de la información, mediante el índice $r$ de Pearson y la eficiencia mediante la siguiente formula:

$$
e=\frac{M r * t p}{M p * T r}
$$

\section{Donde}

$\mathrm{Mr}=$ metas realizadas

$\mathrm{Tp}=$ tiempo programado

$\mathrm{Mp}=$ metas programadas

$\mathrm{Tr}=$ tiempo realizado

\section{Población y Muestra}

Para el cálculo de la muestra se tomará como base la población productora de quinua asociada y no asociada de COPROBICH representado en la tabla 1.
Tabla 1. Población y muestra

\begin{tabular}{cc}
\hline Familias Asociados & Familias No asociados \\
\hline 541 & 745 \\
\hline
\end{tabular}

El cálculo de la muestra se realizará con la fórmula para poblaciones finitas (Gabaldón, 1980), tomando de la COPROBICH (MAGAP, 2017) que en total son de 1.286 familias.

$$
n=\frac{\mathrm{N} \sigma^{2} Z^{2}}{(N-1) e^{2}+\sigma^{2} Z^{2}}
$$

Donde

$\mathrm{N}=$ Población

$\sigma=$ Desviación estándar

$\mathrm{Z}=$ Nivel de Confianza

$\mathrm{e}=$ Error muestral 
Se aplicaon las siguientes consideraciones:

Desviación estándar: 0,5.

Nivel de confianza: 95\% correspondiente a 1,96.

Error muestral: $5 \%$ equivalente a 0,05

$$
\begin{gathered}
n=\frac{1286 *(0,5)^{2} *(1,96)^{2}}{(1285) *(0,05)^{2}+(0,5)^{2} *(1,96)^{2}} \\
n=\frac{1235,0744}{4,1729} \\
n=295,975077
\end{gathered}
$$$$
=296
$$

\section{RESULTADOS}

Resumen de resultados de encuesta de los indicadores de socioeconómicos tanto de asociados y no asociados (Tabla 2).

Resumen de la entrevista realizada a directivos de COPROBICH (Figura 2).

\section{Análisis de Eficiencia}

Medida en que los recursos / insumos (fondos, tiempo, etc.) se han convertido económicamente en productos (output) del proyecto. Se asocia con los componentes del proyecto. Estos datos fueron obtenidos en la entrevista directa con los presentantes de COPROBICH (Tabla 3) para posteriormente determinar su eficiencia

\begin{tabular}{|c|c|c|c|c|c|}
\hline ra 2. Kesumente resulados & & $\operatorname{ados}$ & & iados & \\
\hline & Influye & No influye & Influye & No influye & \\
\hline Ingresos Promedio & 100 & 0 & 71,93 & 28,07 & \\
\hline$\%$ de Acceso a Crédito & 76,8 & 23,2 & 50,29 & 49,71 & Económico \\
\hline Mejora de ingresos & 84,3 & 17,6 & 39,18 & 60,82 & \\
\hline Aseguramiento al IESS & 39,67 & 60,33 & 7,02 & 92,98 & Seguridad y \\
\hline Mejora en alimentación & 64,16 & 32,64 & 35,87 & 64,13 & salud \\
\hline Tipo de construcción & 6,61 & 93,6 & 3,51 & 96,49 & \\
\hline Tipo de piso & 17,36 & 70,4 & 7,02 & 84,8 & Vivianda \\
\hline Tipo de techo & 19,83 & 80,8 & 21,05 & 78,95 & vivienda \\
\hline Servicios & 42,84 & 58,53 & 33,24 & 66,76 & \\
\hline Tenencia de la tierra & 75,2 & 24,8 & 59,65 & 40,35 & \\
\hline Regadío & 2,4 & 97,6 & 1,17 & 98,83 & Productivo y \\
\hline Producción de quinua & 90 & 10 & 30 & 70 & social \\
\hline Producción orgánica & 97,52 & 1,6 & 47,95 & 52,05 & \\
\hline Promedio & 56.07 & 43,93 & 32,00 & 68,00 & \\
\hline
\end{tabular}
en el proyecto.

Tabla 2. Resumen de resultados de encuesta para asociados Asociado

Tabla 3. Representación de la Eficiencia

\begin{tabular}{cccc}
\hline $\begin{array}{c}\text { Número de actividades } \\
\text { programadaspara el } \\
\text { año }\end{array}$ & $\begin{array}{c}\text { Tiempo de realización } \\
\text { de las actividades } \\
\text { programadas }\end{array}$ & $\begin{array}{c}\text { Número de activid ad es } \\
\text { realizad as en el año }\end{array}$ & $\begin{array}{c}\text { Tiempo de realizació } \\
\text { de hs activid ad es } \\
\text { realizad as }\end{array}$ \\
\hline 12 & $\begin{array}{c}12 \\
\quad e=\frac{10 * 12}{12 * 12}\end{array}$ \\
\hline
\end{tabular}

\section{Análisis de Efectividad}

Media en la que se lograron o se espera lograr, los objetivos del proyecto. Se asocia al propósito y los fines directos (Cuantificación de participantes), en donde el programado es lo estimado y lo ejecutado es cuanto se invirtió (Tabla 4).

\section{Análisis de Impactos}

Cambios positivos y negativos primarios y secundarios producidos directa e indirectamente por un proyecto. Se asocia a los fines de un proyecto (Cuantificación productiva) (Tabla 5). 
Figura 2. Resumen de la entrevista por partes de los directivos de COPROBICH

\begin{tabular}{|c|c|c|c|c|c|c|}
\hline ACTIVIDAD & FUENTE DE & EFICIENCIA & EFICACIA & MPACTO & SOSTENIBILIDAD & PERTINENCIA \\
\hline (Descripción) & $\begin{array}{l}\text { VERIFICACION } \\
\text { (Documentación Base) }\end{array}$ & $\begin{array}{l}\text { Medida en que los } \\
\text { recursos / insumos } \\
\text { (fondos, tiempo, } \\
\text { etc.) se han } \\
\text { convertido } \\
\text { económicamente } \\
\text { en productos } \\
\text { (output) del } \\
\text { proyector Se } \\
\text { asocia con los } \\
\text { componentes del } \\
\text { proyecto. } \\
\text { (Cuantificación } \\
\text { económica) }\end{array}$ & $\begin{array}{l}\text { Media en la que } \\
\text { se lograron o se } \\
\text { espera lograr, } \\
\text { los objetivos del } \\
\text { proyecto. Se } \\
\text { asocia al } \\
\text { propósito y los } \\
\text { fines directos. } \\
\text { (Cuantificación } \\
\text { de } \\
\text { participantes) }\end{array}$ & $\begin{array}{l}\text { Cambios positivos y } \\
\text { negativos primarios y } \\
\text { secundarios } \\
\text { producidos directa e } \\
\text { indirectamente por un } \\
\text { proyecto. Se asocia a } \\
\text { los fines de un } \\
\text { proyecto } \\
\text { (Cuantificación } \\
\text { productiva) }\end{array}$ & $\begin{array}{l}\text { Continuidad en la } \\
\text { generación de beneficios } \\
\text { de un proyecto. Se asocia } \\
\text { con el mantenimiento de } \\
\text { la capacidad de provisión } \\
\text { de bienes y servicios y el } \\
\text { uso o utilización de éstos } \\
\text { por parte de los } \\
\text { beneficiarios. } \\
\text { (Cuantificación } \\
\text { temporal) }\end{array}$ & $\begin{array}{l}\text { Medida en la que los } \\
\text { objetivos de un } \\
\text { proyecto } \\
\text { coherentes con las } \\
\text { necesidades de los } \\
\text { beneficiarios, los } \\
\text { contextos local, } \\
\text { municipal y } \\
\text { departamental, y con } \\
\text { las políticas públicas } \\
\text { sectoriales del pais. } \\
\text { (Ajuste a Planes de } \\
\text { Desarrollo) }\end{array}$ \\
\hline $\begin{array}{l}\text { Contratación de personal } \\
\text { técnico y administrativo } \\
\text { para administración del } \\
\text { proyecto. }\end{array}$ & $\begin{array}{l}\text { Presupuesto } \\
\text { COPROBICH } \\
\text { IEPS 2017 }\end{array}$ & $\begin{array}{l}\text { Inversión: } \\
\text { \$25.200 } \\
\text { Ejecución: } \\
\$ 25.200 \\
(100 \%)\end{array}$ & $\begin{array}{l}\text { Aprobación del } \\
\text { presupuesto: } \\
\text { Participan el } \\
56 \% \text { de socios. }\end{array}$ & $\begin{array}{l}\text { Resultados del trabajo } \\
\text { mediante evaluación } \\
\text { del personal } \\
\text { contratado: } 95 \%\end{array}$ & $\begin{array}{l}\text { Resultado de continuidad } \\
\text { del trabajo del personal } \\
\text { contratado: } 100 \%\end{array}$ & $\begin{array}{l}\text { Totalmente adecuado } \\
\text { al Plan de Desarrollo } \\
\text { por la necesidad de } \\
\text { personal } \\
\text { administrativo de } \\
\text { sustento y personal } \\
\text { técnico asesor. }\end{array}$ \\
\hline $\begin{array}{l}\text { Implementación del } \\
\text { laboratorio de saponina y } \\
\text { otros. }\end{array}$ & $\begin{array}{l}\text { Presupuesto } \\
\text { COPROBICH } 2017\end{array}$ & $\begin{array}{l}\text { Inversión: } \\
\text { \$41.871 } \\
\text { Ejecución: } \\
\text { \$41.871 } \\
(100 \%)\end{array}$ & $\begin{array}{l}\text { Aprobación del } \\
\text { presupuesto: } \\
\text { Participan el } \\
56 \% \text { de socios. }\end{array}$ & $\begin{array}{l}\text { Instalación completa } \\
\text { del laboratorio al } \\
100 \%\end{array}$ & $\begin{array}{l}\text { Laboratorio en } \\
\text { funcionamiento al } 100 \%\end{array}$ & $\begin{array}{l}\text { Totalmente adecuado } \\
\text { al Plan de Desarrollo } \\
\text { por la necesidad de } \\
\text { contar con un } \\
\text { laboratorio para } \\
\text { mejorar la calidad del } \\
\text { producto. }\end{array}$ \\
\hline $\begin{array}{l}\text { Adquisición de equipos de } \\
\text { Iaboratorio. }\end{array}$ & $\begin{array}{l}\text { Presupuesto } \\
\text { COPROBICH } 2017\end{array}$ & $\begin{array}{l}\text { Inversión: } \\
\text { \$4.589 } \\
\text { Ejecución: } \\
\$ 4.589 \\
(100 \%)\end{array}$ & $\begin{array}{l}\text { Aprobación del } \\
\text { presupuesto: } \\
\text { Participan el } \\
56 \% \text { de socios. }\end{array}$ & $\begin{array}{l}\text { Compras ejecutadas } \\
\text { por portal de compras } \\
\text { públicas con } \\
\text { efectividad del } 100 \%\end{array}$ & $\begin{array}{l}\text { Materiales adquiridos } \\
\text { certificados con calidad } \\
\text { adecuada al } 100 \% \text { de las } \\
\text { especificaciones }\end{array}$ & $\begin{array}{l}\text { Totalmente adecuado } \\
\text { al Plan de Desarrollo } \\
\text { por la necesidad de } \\
\text { implementar un } \\
\text { laboratorio para } \\
\text { mejorar la calidad del } \\
\text { producto. }\end{array}$ \\
\hline $\begin{array}{l}\text { Contratación de estación } \\
\text { de control de plagas y } \\
\text { roedores. }\end{array}$ & $\begin{array}{l}\text { Presupuesto } \\
\text { COPROBICH } 2017\end{array}$ & $\begin{array}{l}\text { Inversión: } \\
\text { \$4.014 } \\
\text { Ejecución: } \\
\$ 4.014 \\
(100 \%)\end{array}$ & $\begin{array}{l}\text { Aprobación del } \\
\text { presupuesto: } \\
\text { Participan el } \\
56 \% \text { de socios. }\end{array}$ & $\begin{array}{l}\text { Estación de control en } \\
\text { funcionamiento al } \\
100 \%\end{array}$ & $\begin{array}{l}\text { Trabajo permanente de la } \\
\text { estación con } 100 \% \text { de } \\
\text { efectividad en el trabajo. }\end{array}$ & $\begin{array}{l}\text { Importante por la } \\
\text { relación entre la } \\
\text { mejora de la } \\
\text { productividad y la } \\
\text { prevención de plagas. }\end{array}$ \\
\hline $\begin{array}{l}\text { Contratación de estudios } \\
\text { de mercado para la quinua } \\
\text { y sus derivados. }\end{array}$ & $\begin{array}{l}\text { Presupuesto } \\
\text { COPROBICH } 2017\end{array}$ & $\begin{array}{l}\text { Inversión: } \\
\text { \$12.000 } \\
\text { Ejecución: } \\
\$ 12.000 \\
(100 \%)\end{array}$ & $\begin{array}{l}\text { Aprobación del } \\
\text { presupuesto: } \\
\text { Participan el } \\
56 \% \text { de socios. }\end{array}$ & $\begin{array}{l}\text { Entrega de informe } \\
\text { completo }(100 \%)\end{array}$ & $\begin{array}{l}\text { Resultados del informe } \\
\text { válidos para el año } \\
\text { completo. }\end{array}$ & $\begin{array}{l}\text { Necesario por contar } \\
\text { con mercados fijos y } \\
\text { seguros para la } \\
\text { producción. }\end{array}$ \\
\hline $\begin{array}{l}\text { Restauración de la planta } \\
\text { procesadora que quinua. }\end{array}$ & $\begin{array}{l}\text { Presupuesto } \\
\text { COPROBICH } 2017\end{array}$ & $\begin{array}{l}\text { Inversión: } \\
\text { \$78.248 } \\
\text { Ejecución: } \\
\$ 80.063 \\
(-2,32 \% \\
\text { déficit) }\end{array}$ & $\begin{array}{l}\text { Aprobación del } \\
\text { presupuesto: } \\
\text { Participan el } \\
56 \% \text { de socios. }\end{array}$ & $\begin{array}{l}\text { Planta procesadora } \\
\text { funcional en un } 100 \%\end{array}$ & $\begin{array}{l}\text { Planta funcional al menos } \\
\text { para } 5 \text { años. }\end{array}$ & $\begin{array}{l}\text { Actividad necesaria } \\
\text { para mejorar la } \\
\text { productividad } \\
\text { industrial de la } \\
\text { quinua. }\end{array}$ \\
\hline $\begin{array}{l}\text { Auditoria del ARCSA- } \\
\text { MSP }\end{array}$ & $\begin{array}{l}\text { Gastos operativos no } \\
\text { programados }\end{array}$ & $\begin{array}{l}\text { Inversión: } \\
\$ 1.000 \\
\text { Ejecución: } \\
\$ 1.000 \\
(100 \%)\end{array}$ & $\begin{array}{l}\text { Aprobación de } \\
\text { gastos comisión } \\
\text { permanente } \\
\text { financiera. }\end{array}$ & $\begin{array}{l}\text { Informe de auditoria } \\
\text { útil para evaluación } \\
\text { interna y y } \\
\text { retroalimentación. }\end{array}$ & $\begin{array}{l}\text { Informe útil al menos para } \\
\text { un año. }\end{array}$ & $\begin{array}{l}\text { Actividad } \\
\text { fundamental prevista } \\
\text { en la ley y útil para la } \\
\text { administración. }\end{array}$ \\
\hline $\begin{array}{l}\text { Implementación de la } \\
\text { marca mediante impresos. }\end{array}$ & $\begin{array}{l}\text { Presupuesto } \\
\text { COPROBICH } 2017\end{array}$ & $\begin{array}{l}\text { Inversión: } \\
\$ 7.296 \\
\text { Ejecución: } \\
\$ 7.296 \\
(100 \%)\end{array}$ & $\begin{array}{l}\text { Aprobación del } \\
\text { presupuesto: } \\
\text { Participan el } \\
56 \% \text { de socios. }\end{array}$ & $\begin{array}{l}\text { En relación con el } \\
\text { replanteo de la marca } \\
\text { y la captación de } \\
\text { nuevos mercados. }\end{array}$ & $\begin{array}{l}\text { Material impreso para un } \\
\text { año. }\end{array}$ & $\begin{array}{l}\text { Acción útil para la } \\
\text { ampliación de } \\
\text { mercados a nivel } \\
\text { local e intemacional. }\end{array}$ \\
\hline $\begin{array}{l}\text { Capacitación } \\
\text { fortalecimiento } \\
\text { organizacional. }\end{array}$ & $\begin{array}{l}\text { Gastos operativos no } \\
\text { programados }\end{array}$ & $\begin{array}{l}\text { Inversión: } \\
\text { \$1.148 } \\
\text { Ejecución: } \\
\$ 1.148 \\
(100 \%)\end{array}$ & $\begin{array}{l}\text { Cabecillas, } \\
\text { comisión de } \\
\text { vigilancia, } \\
\text { directorio, } \\
\text { equipo técnico y } \\
\text { trabajadores. } \\
\text { Asistencia del } \\
90 \% \\
\text { programado. }\end{array}$ & $\begin{array}{lr}\begin{array}{l}\text { Promueve } \\
\text { integración de la }\end{array} \\
\begin{array}{l}\text { socios en los } \\
\text { actividades } \\
\text { productivas }\end{array} \\
\text { COPROBICH. de } \\
\end{array}$ & $\begin{array}{l}\text { Motivación al menos para } \\
6 \text { meses en función de la } \\
\text { participación activa de los } \\
\text { socios en las acciones de } \\
\text { la Corporación. }\end{array}$ & $\begin{array}{l}\text { Actividad } \\
\text { relacionada con las } \\
\text { organizaciones de la } \\
\text { Economia Popular y } \\
\text { Solidaria. }\end{array}$ \\
\hline
\end{tabular}

Tabla 4. Representación de la efectividad

\begin{tabular}{cccc}
\hline $\begin{array}{c}\text { Resumen de indicadores de } \\
\text { resultados }\end{array}$ & Programado & Ejecutado & \% de efectividad \\
\hline Maquinaria y equipamiento & 136833 & 135018 & $98,67 \%$ \\
Comercialización & 31866 & 17832 & $55,96 \%$ \\
Fortalecimiento organizativo & 1305 & 1305 & $100 \%$ \\
Equipo técnico & 25200 & 25200 & $100 \%$ \\
\% de Efectividad & & & $\mathbf{8 8 , 6 6 \%}$ \\
\hline
\end{tabular}


Tabla 5. Cálculo del impacto

\begin{tabular}{cccc}
\hline & Asociados $(\mathbf{x})$ & No Asociados $(\mathbf{y})$ & Impactos \\
\hline Ingresos Promedio & 100 & 71,93 & 28,07 \\
\% de Acceso a Crédito & 76,8 & 50,29 & 26,51 \\
Mejora de ingresos & 82,4 & 39,18 & 43,22 \\
Aseguramiento al IESS & 39,67 & 7,02 & 32,65 \\
Mejora en alimentación & 67,36 & 35,87 & 31,49 \\
Tipo de construcción & 6,4 & 3,51 & 2,89 \\
Tipo de piso & 29,6 & 15,2 & 14,4 \\
Tipo de techo & 19,2 & 21,05 & $-1,85$ \\
Servicios & 41,47 & 33,24 & 8,23 \\
Tenencia de la tierra & 75,2 & 59,65 & 15,55 \\
Regadío & 2,4 & 1,17 & 1,23 \\
Producción de quinua & 90 & 30 & 60 \\
Producción orgánica & 98,4 & 47,95 & 50,45 \\
\hline
\end{tabular}

\section{Validez de la información}

La validez de los datos obtenidos, de acuerdo con el índice r de Pearson se sitúa en el 92,863061\%.

Se comprueba que con un $92,86 \%$ de certeza, existe evidencia de mejora socioeconómica entre los asociados a COPROBICH.

Tabla 6. Proyección de datos

\begin{tabular}{ccccc}
\hline Indicadores de impacto & Asociados (x) & No asociados (y) & Impacto & Proyección (b) \\
\hline Ingresos Promedio & 100 & 71,93 & 28,07 & 55,87 \\
\% de Acceso a crédito & 76,8 & 50,29 & 26,51 & 43,27 \\
Mejora de ingresos & 82,4 & 39,18 & 43,22 & 46,31 \\
Aseguramiento al IESS & 39,67 & 7,02 & 32,65 & 23,10 \\
Mejora en alimentación & 67,36 & 35,87 & 31,49 & 38,14 \\
Tipo de construcción & 6,4 & 3,51 & 2,89 & 5,02 \\
Tipo de piso & 29,6 & 15,2 & 14,4 & 17,62 \\
Tipo de techo & 19,2 & 21,05 & $-1,85$ & 11,97 \\
Servicios & 41,47 & 33,24 & 8,23 & 24,07 \\
Tenencia de la tierra & 75,2 & 59,65 & 15,55 & 42,40 \\
Regadío & 2,4 & 1,17 & 1,23 & 2,85 \\
Producción de quinua & 90 & 30 & 60 & 50,44 \\
Producción orgánica & 98,4 & 47,95 & 50,45 & 55,00 \\
\hline
\end{tabular}

\section{Sostenibilidad}

Continuidad en la generación de beneficios de un proyecto. Se asocia con el mantenimiento de la capacidad de provisión de bienes y servicios y el uso o utilización de éstos por parte de los beneficiarios.

La sostenibilidad ubica al proyecto con el 100\% debido a la naturaleza del proyecto por que potenciar las capacidades de producción teniendo como objetivo final el de general una soberanía alimentaria que

\section{Proyección de datos}

Para la proyección de datos se ha empleado la fórmula de pronóstico de regresión lineal de tendencia que ayudara a medir, el indicar o los indicadores en que se debe tomar acciones, ante la toma de decisiones (Tabla 6).

fortalezca el desarrollo rural.

\section{IV.DISCUSIÓN}

Hay estudios de evaluación Ex-post parecidos al realizado, un ejemplo es la "Evaluación Ex-post de proyectos de desarrollo de fincas agroforestales en zona de pre montaña" realizado en Cuba, llegaron a concluir que la aplica un procedimiento lógicamente estructurado, que permite realizar, dentro del ciclo de vida de 
los proyectos de inversión en la agricultura de montaña y atendiendo a la temporalidad de la acción evaluadora, una apreciación de la gestión del proceso inversionista. El proyecto no fue ejecutado como se planificó, fue subcosteado en un 59\% lo que se refleja consecuentemente en los indicadores de eficiencia. De forma general el proyecto muestra en la evaluación de la gestión resultados favorables que permiten calificarlo de sostenible y pertinente (Varela, 2015)

Un estudio realizado en Colombia "Evaluación expost del Proyecto Contribución a la seguridad alimentaria a las familias vulnerables de Antioquia" determino que los promedios de producción de los emprendimientos registran un incremento en casi todos los rubros incluidos en los emprendimientos, pero no ofrecen evidencia estadística de incrementos significativos. Las réplicas de las acciones asociativas se produjeron en cerca del $17 \%$ de los productores participantes. Los promedios de los precios recibidos por los productores asociados a los emprendimientos son también mayores después de los esfuerzos del proyecto para conectar los productores a los mercados, pero con la excepción de las hortalizas, no son estadísticamente diferentes a los precios que recibían los productores antes de las acciones del proyecto. Otro dato a destacar del proyecto es que la participación de hombres y mujeres no es igual en las asociaciones y se presentan variaciones, según el tipo de emprendimiento. En total, la participación de las mujeres es copiosa y ligeramente superior al $42 \%$, mostrando que el proyecto ha abierto oportunidades a la participación de las mujeres (FAO, 2016)

Otro estudio realizado en Ecuador "Evaluación ex post socioeconómico al proyecto Madre Teresa de Calcuta, provincia de Chimborazo, cantón Cumanda" llego a la conclusión de que a través de este proyecto se evidencia un impacto socioeconómico positivo ya que por medio de este se ha logrado dar mejor calidad de vida a los involucrados, eliminado la migración en la localidad. Se observa que los indicadores tuvieron resultados favorables por lo que los actores lograran obtener buenos rendimientos mensuales sumados a una mejora continua de la calidad de vida. Con la implementación de este proyecto se ha podido concientizar a la gente para el cuidado del medio ambiente por lo que se ha visto una buena práctica de utilización de las excretas de los cerdos (Chávez, 2016).

La ayuda de una evaluación Ex -post en proyectos relacionados a la agricultura y ganadería entre otros, es fundamental ya que nos da un aporte significativo en la toma de decisiones de ciertos proyectos que pueden generar beneficios a sus asociados siendo identificando claramente en los proyectos antes investigados, que sus aportes son positivos ayudan y fomentan la socioeconómica en las familias asociada con una actividad que ellos continuamente realizan que es la agricultura, que más satisfactorio como es el caso de la Quinua que es una alimento rico en proteína, además que las familias cuente con ingresos económicos y puedan mejor su estilo de vida.

\section{CONCLUSIONES}

En la evaluación socioeconómica de los socios de COPROBICH antes de la implementación del proyecto quinua, se tomaron datos de la encuesta socioeconómica realizada en el 2017 a asociados y no asociados, de esta comparación se pudo determinar que en los indicadores principales tomados como base de análisis existen diferencias, aunque no tan sustanciales.

Concretamente, los ingresos provenientes del proyecto quinua son mejores en los asociados en un $28,07 \%$ respecto a los no asociados, el acceso a créditos en un $26,51 \%$, la percepción de mejora en ingreso es un $19,7 \%$ más alta en los asociados, el nivel de seguridad social es $32,65 \%$ mayor que los no asociados, las mejoras en habitabilidad varían entre un 2,89, y la organicidad de la producción se elevó en un 50,42\%. Los demás indicadores registran incrementos bajos no representativos para el desarrollo socioeconómico de la población, por lo que la influencia del proyecto en la calidad de vida, es comprobado, pero no en niveles demasiado elevados.

Se establece un nivel de cumplimiento de validez del $92,86 \%$, basados en el índice de Pearson, mientras que 
la eficiencia alcanza el $83,33 \%$ y la efectividad un

$88,66 \%$. La pertinencia y la sostenibilidad se ubican al $100 \%$ debido a la naturaleza del proyecto. Se determina que el bajo nivel de impacto se debe a que las actividades del proyecto COPROBICH se centran más en el desarrollo productivo y distributivo que en el desarrollo de los asociados, es decir, los proyectos generados no revierten su influencia hacia las bases de la organización, sino hacia el desempeño corporativo.

Las acciones desarrolladas en el proyecto quinua por la Corporación de Productores y Comercializadores Orgánicos "Bio Taita Chimborazo" (COPROBICH) en el cantón Colta impactaron positivamente en la situación socioeconómica de las familias asociadas.

\section{REFERENCIAS BIBLIOGRÁFICAS}

Chávez, X. R. 2016. Evaluación ex-post socioeconómico al proyecto madre Teresa de Calcuta, provincia de Chimborazo, cantón Cumandá, periodo 2014-2015. Tesis de Grado. Escuela Superior Politecnica de Chimborazo. Chimborazo (Ecuador).

Contreras, E. 2014. Evaluación de Inversiones Públicas: enfoques alternativos y su aplicabilidad para Chile. Santiago de Chile (Chile): CEPAL

COPROBICH. 2017. ¿Quiénes somos? http://coprobich.com/ (Consultada el 30 de marzo de 2018)

FAO (Organización de las Naciones Unidas para la Alimentación y Agricultura). 2015. Rurallnvest: para mejorar las inversiones y los medios de vida. http://www.fao.org/inaction/rural-invest/es/ (Consultada el 20 de agosto de 2018)

FAO (Organización de las Naciones Unidas para la Alimentación y Agricultura). 2016. Evaluación ex-post del Proyecto Contribución a la seguridad alimentaria de las familias vulnerables de Antioquia. Informe técnico. Roma (Italia)

Fundación Ethos. 2015. Índice Ethos de Pobreza. https://www.ethos.org.mx/ (Consultada el 25 de septiembre de 2017)

Gabaldón, N. 1980. Algunos conceptos de muestreo. Caracas (Venezuela): Universidad Central de Venezuela.

GADPC (Gobierno Provincial de Chimborazo). 2017. Fortalecimiento del proceso de producción y diversificación de la Quinua orgánica en la planta procesadora de COPROBICH. Riobamba. Informe técnico. Riobamba (Ecuador).

MAGAP (Ministerio de Agricultura y Ganadería). 2017. 2017, año clave para Ecuador en exportación de quinua. https://www.agricultura.gob.ec/2017-anoclave-para-ecuador-en-exportacion-dequinua/ (Consultada el 29 de abril de 2018)

Medina, H. 2014. Evaluación ex post en la gestión de proyectos PIP. Ciudad de México (México): UNAM.

Montero, A. 2016. Rendimientos de la Quinua en el Ecuador 2016. Informe técnico. Quito (Ecuador).

Navarro, H., K. King, E. Ortegón, y J. F. Pacheco. 2016. Pauta metodológica de evaluación de impacto ex ante y ex post de programas sociales de lucha contra la pobreza. Santiago de Chile (Chile): CEPAL.

Varela, M. C. M. 2015. "Evaluación Ex-post de proyectos de desarrollo de fincas agroforestales en zona de pre montaña." Universidad y Sociedad 7 (3): 6-11. 


\section{APÉNDICE 1}

\section{ENCUESTA}

ESCUELA SUPERIOR POLITÉCNICADE CHIMBORAZO

ENCUESTA SOCIOECONÓMICA

$N^{\circ}$ DE ENCUESTA:

Fecha de la encuesta:

Ubicación:

1.- ASPECTO SOCIOECONÓMICO

1.1.- Información personal

1) Persona entrevistada: Padre

Madre

Otros: Parentesco:

2) Edad:

3) Nivel educativo: Sin instrucción:

Básica:

Media:

Tecnología:

Tercer nivel:

Cuarto nivel:

4) Estado civil: Soltero/a:

Casado/a:

Unión Libre:

Viudo/a:

Divorciado/a:

5) Ocupación: A $\overline{\text { gricultor: }}$

Jornalero:

Empleado:

Otro:

¿Cuál?:

1.2.- Información familiar:

6) ¿Tiene hijos? $\mathrm{Si}$

7) Detalle de hijos:

Número de hijo Edad Escolaridad Sostenimiento Aporte de COPROBICH en educación

1.3.- Información económica:

8) ¿Cuál es su fuente principal de ingresos?

Agricultura:

Ganadería:

Jornalero:

Otras: ¿Cuál?

9) ¿Cuál es su ingreso aproximado anual en la producción de quinua, y su ingreso de hace un año? Actual: Hace un año:

10) ¿Ha tenido acceso a créditos productivos? $\mathrm{Si}$ :

11) ¿En qué tipo de instituciones financieras? No:

Bancos:

Cooperativas:

Financieras:

Usureros:

12) ¿Considera que COPROBICH ha mejorado su ingreso familiar? Si:

No:

13) ¿Qué herramientas ha recibido de parte de COPROBICH para la producción de quinua?

HERRAMIENTAS CANTIDAD COSTO TOTALAzadón

Pala

Rastrillo

Carretilla

Bomba de fumigar

Pico

sistema de Riego

Tractor

Yunta

14) Considera usted que sus ingresos, respecto al año anterior son:

Mayores:

Iguales:

Menores:

15) ¿En qué aspectos considera que COPROBICH ha influido en su economía?

Capacitación:

Asesoría:

Acceso a créditos:

Equipamiento: 
Técnicas nuevas:

Certificaciones:

Mercados seguros:

Semillas:

Riego:

Abonos:

Cuidado del entorno:

16) ¿Participa usted en los siguientes eventos de COPROBICH? Sesiones:

Rendición de cuentas:

Beneficios:

Control interno:

1.4.- Seguridad social y alimentación:

17) ¿Tiene algún seguro de salud? Si: No:

18) ¿En qué institución?

19) ¿Tiene su familia algún tipo de seguro de salud? Si:

20) ¿En qué institución?

21) ¿Ha empleado usted el seguro de salud en el último año? Si:

22) ¿En qué forma aporta COPROBICH en la salud de su familia?

23) ¿Con qué frecuencia consume usted los siguientes tipos de alimentos?

Diario Semanal Mensual

Proteína animal (Carnes, huevos)

Proteína vegetal (Chocho, quinua, fréjol, avena)

Carbohidratos (papas, arroz, fideos)

Vegetales (Tomate, lechuga, rábano, remolacha)

Grasas (Aceite, manteca, mantequilla)

1.5.- Vivienda:

24) ¿Qué tipo de vivienda tiene?

Casa

Finca

Quinta

25) ¿Cuál es el material de construcción?

Hormigón:

Adobe:

Mixta:

26) ¿De qué son los pisos de su casa?

Cemento:

Madera:

Cerámica:

Tierra:

27) ¿De qué material es su techo?

Zinc

Fibrocemento:

Paja:

1.6.- Servicios:

28) ¿Cuáles de estos servicios posee?

29) ¿Cuál es el monto de gastos por servicios actual?

30) ¿Cuál fue el monto de gastos por servicios hace un año?

31) ¿De qué manera ayuda COPROBICH en los gastos de vivienda?

\section{ASPECTO PRODUCTIVO}

32) ¿La tierra que cultiva es propia? $\mathrm{Si}$ : No:

33) Si la tierra que cultiva no es propia: ¿Qué forma de tenencia posee?

Arrienda:

Comparte:

Otras formas:

¿Cuáles?

34) ¿Tiene sistema de regadío? $\overline{\mathrm{Si}}$

35) ¿Está asociado a COPROBICH? Si:

36) ¿Cuánto produce anualmente en quinua?

37) ¿Cuánto produjo en quinua hace un año?

38) Si es asociado ¿Qué beneficios ha obtenido de COPROBICH?

39) ¿Cree usted que COPROBICH ha mejorado su calidad de vida? Si:

40) ¿En qué forma?

41) ¿Ha recibido apoyo de otras organizaciones en la producción de quinua? Si: No:

42) ¿Emplea usted productos químicos en la producción de quinua? Si: 


\section{APÉNDICE 2}

\section{ENTREVISTA \\ ESCUELA SUPERIOR POLITÉCNICA DE CHIMBORAZO ENTREVISTA A DIRECTIVOS DE COPROBICH}

\section{A. PERTINENCIA}

Medida en la que los objetivos de un proyecto son coherentes con las necesidades de los beneficiarios, los contextos local, municipal y departamental, y con las políticas públicas sectoriales del país. (Ajuste a Planes de Desarrollo) 1. ¿El objetivo general del proyecto quinua de COPROBICH fue validado con las políticas del Estado y del sector agrícola local? ¿Qué aspectos se consideraron para esta coordinación?

2. ¿En qué forma se relacionan las acciones del proyecto quinua de COPROBICH con el objetivo de la organización y las políticas estatales y del sector?

3. ¿De qué manera el proyecto quinua de COPROBICH ha considerado las necesidades de sus asociados en las actividades desarrolladas?

4. ¿De qué manera se han identificado los riesgos propios de las actividades del proyecto quinua desarrolladas por COPROBICH el en último año?

\section{B. EFICIENCIA}

Medida en que los recursos / insumos (fondos, tiempo, etc.) se han convertido económicamente en productos (output) del proyecto. Se asocia con los componentes del proyecto. (Cuantificación económica)

- ¿Las actividades desarrolladas por el proyecto quinua desarrollado por COPROBICH se han desarrollado con la participación de todos sus asociados? ¿En qué porcentaje lo han hecho?

6. ¿Las actividades programadas se realizaron en los plazos previstos por la planificación? ¿Se han ejecutado todas las actividades programadas?

7. ¿Las actividades se realizaron de acuerdo a lo presupuestado originalmente? ¿Qué variaciones financieras hubo?

8. Fórmula de la eficiencia: $\mathrm{e}=\mathrm{Mr} * \mathrm{Tp} / \mathrm{Mp} * \mathrm{Tr}(\mathrm{Mr}=$ metas realizadas; $\mathrm{Tp}=$ tiempo programado; $\mathrm{Mp}=$ metas programadas; $\mathrm{Tr}=$ Tiempo realizado)

\section{EFICACIA}

Media en la que se lograron o se espera lograr, los objetivos del proyecto. Se asocia al propósito y los fines directos. (Cuantificación de participantes)

9. ¿En qué medida se han cumplido los objetivos de las actividades desarrolladas por COPROBICH durante el último año?

10. ¿En qué medida se han cumplido las actividades previstas por COPROBICH relacionadas con el proyecto quinua?

¿Cuántos asociados tiene actualmente COPROBICH en relación con el proyecto quinua?

12. ¿Cuántos asociados han participado del $100 \%$ de actividades realizadas en el proyecto quinua por COPROBICH?

13. ¿Cuál es la rentabilidad social de las acciones emprendidas por COPROBICH en el proyecto quinua?

\section{IMPACTO}

Cambios positivos y negativos primarios y secundarios producidos directa e indirectamente por un proyecto. Se asocia a los fines de un proyecto (Cuantificación productiva)

14. ¿Hasta qué punto se han cumplido con los impactos previstos en el proyecto quinua?

15. ¿En qué aspectos se han centrado los impactos de las actividades del proyecto quinua?

16. ¿Qué impactos negativos considera que han generado las actividades desarrolladas por el proyecto quinua?

17. ¿Considera usted que el proyecto propicia la generación de empleos en la sociedad? ¿De qué manera?

18. ¿De qué manera aporta el proyecto a la salud de los asociados?

19. Qué modelo organizativo adopta la corporación frente a sus asociados?

20. ¿De qué manera y cómo se monitoriza la mejora económica de los asociados a COPROBICH?

21. ¿De qué manera se ha cuantificado el impacto ambiental de la corporación?

22. ¿Qué aspectos culturales se han modificado en la población con la implementación del proyecto Quinua?

23. ¿De qué manera se ha reforzado la organización comunitaria de los asociados a COPROBICH?

\section{E.SOSTENIBILIDAD}

Continuidad en la generación de beneficios de un proyecto. Se asocia con el mantenimiento de la capacidad de provisión de bienes y servicios y el uso o utilización de éstos por parte de los beneficiarios. (Cuantificación temporal) 24. ¿De qué manera se financian las actividades del proyecto quinua?

25. ¿Qué grado de sostenibilidad tiene el proyecto quinua a través de sus actividades?

26. ¿Qué factores considera que influyen en la sostenibilidad del proyecto quinua?

\section{7. ¿De qué manera mejoraría la sostenibilidad del proyecto quinua?}

\section{GRACIAS POR SU COLABORACIÓN}




\section{APÉNDICE 3}

MATRIZ DE LA ENTREVISTA

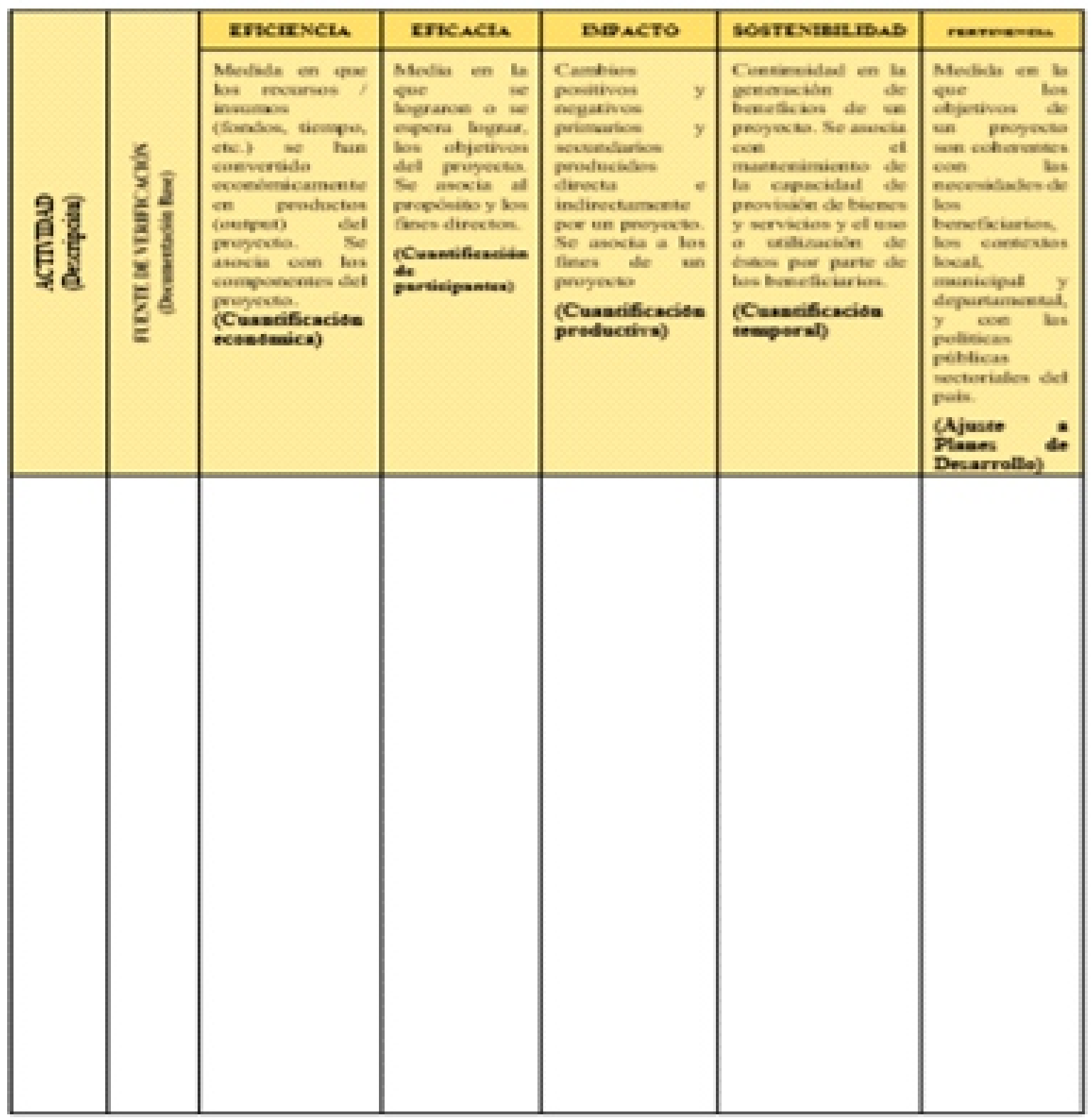

